\title{
Performance Analysis of Indoor Optical Wireless Links
}

\author{
Ram Kumar Sharma \\ ITM University, Sec 23A, Huda City, Gurgaon, India. \\ Hemani Kaushal \\ ITM University, Sec 23A, Huda City, Gurgaon, India. \\ Prabhat Kumar Sharma \\ ITM University, Sec 23A, Huda City, Gurgaon, India.
}

\begin{abstract}
Indoor wireless optical communication is a good alternative to existing mature $R F$ technology. However various challenges in indoor optical wireless technology are due to free space loss, ambient light, and multi path dispersion causing inter symbol interference (ISI). The degradation in performance due to these facts is very much influenced by the channel topology. So in this paper the performance of indoor optical configuration has been analyzed using three types of channel topologies viz., directed (LOS), non-directed (LOS), and multi beam diffused link for various transmitter and receiver design parameters. The analysis has been carried using Optiwave simulation tools.
\end{abstract}

Keywords: Optical wireless communication, BER, Q-factor, NRZ-OOK, LOS, eye diagram, and multi beam diffused channel.

\section{INTRODUCTION}

I ndoor optical wireless (IOW) communication system [1] consists of a transmitter which is generally a light emitting diode (LED) or a laser diode with a wavelength ranging from infrared (IR) to ultraviolet (UV) including visible light spectrum. The receiver is usually a low cost PN/PIN photodetector. The transmitter and the receiver communicate through optical waves over the free space channel. The advantages of optical wireless communication are availability of large $(\mathrm{THz})$ bandwidth, unregulated spectrum, inherent security, low system cost and immunity towards electromagnetic interference [2]. Such links usually adopt to intensity modulation/ direct detection (IM/ DD) and efficient spatial diversity techniques hence preventing multi path fading effects [3]. Further, it is possible to reuse the frequency and make secure communication at physical level, since optical beam does not penetrate walls or opaque objects [4].

Indoor optical wireless systems need to be evolved as a mobile system within indoor environment. So under various channel topologies, the aim is to investigate the performance of the system parameters to achieve higher SNR and lower bit error rate (BER) without increase in the transmitted power by varying the design parameters such as transmitter beam angle at the transmitter

Journal of Technology Management for Growing Economies Vol. 6, No. 1 April 2015 pp. $15-26$

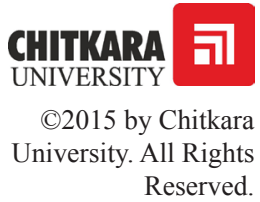


Sharma, R. K. Kaushal, $\mathrm{H}$. Sharma, P. K.

side and field of view (FOV) at receiver side. With this motivation, we in this paper present a simulation based analysis of IOW communication system with receiver mobility.

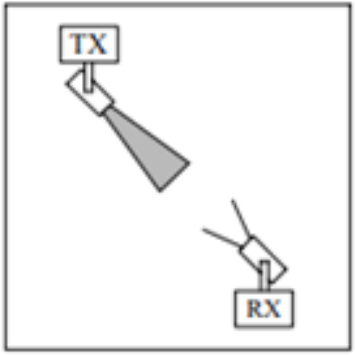

(a)

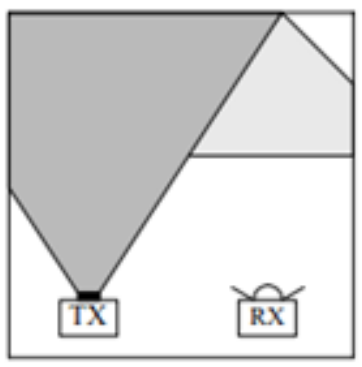

(c)

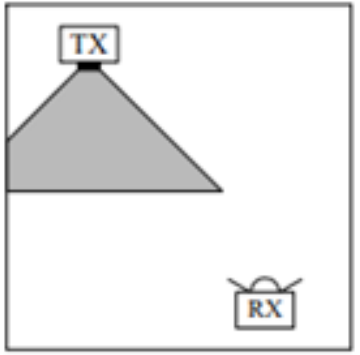

(b)

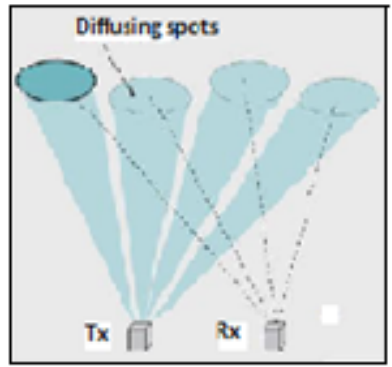

(1)

Figure 1: Link Configurations: (a) directed LOS, (b) non-directed LOS, (c) diffused Link, (d) Multi beam diffused links

Rest of this paper is organized as follows. Section II presents the brief description of various channel topologies. Section III describes the simulation set up. In section IV, the results obtained from Optiwave simulations are expressed. Finally, conclusion is presented in section V.

\section{OPTICAL INDOOR CHANNEL TOPOLOGY}

IOW communication system may employ two types of link topologies [5], depending on the transmitter's beam directionality and receiver's field of view (FOV). First is line of sight (LOS) links, and second is non-LOS link. The LOS links are also of two types, one is directed LOS in which the transmitter and receiver are directed towards each other with narrow transmitter semi beam divergence angle and smaller receiver FOV as shown in Fig. 1(a). The other one is the non directed-LOS link which involves wider beam divergence angle and comparatively large receiver's field of view as 


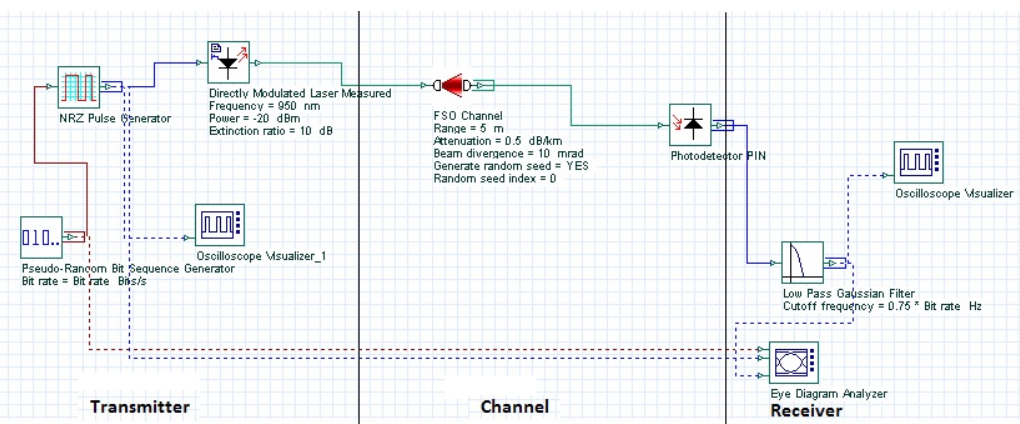

Performance

Analysis of Indoor

Figure 2: Simulation setup for directed \& non-directed LOS

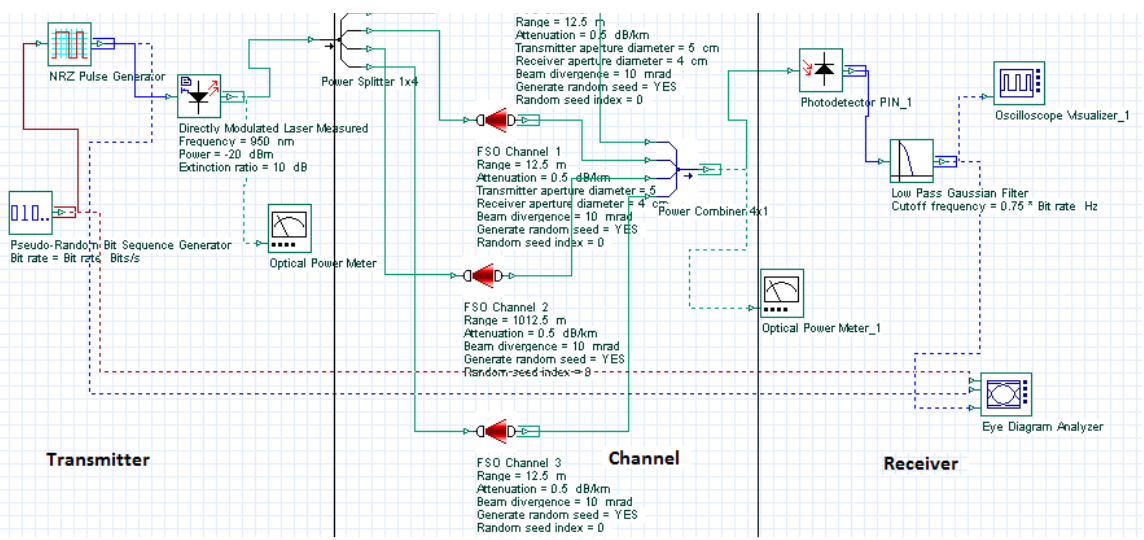

Figure 3: Simulation setup for multi beam diffused topology

expressed in Fig. 1(b). In IOW systems with moving receiver, the non-LOS category is commonly employed as per Fig. 1(c) and Fig. 1(d). The diffused links rely upon the reflected path of light from the walls, ceiling or any other diffusely reflecting surface. The optical signal from transmitter to receiver undergoes temporal dispersion due to such reflections. This causes inter symbol interference (ISI) which is the major impediment to the transmission speed [6]. Further, such links do not get blocked by the obstacles within the room as compared to LOS links. The diffused channel mechanism (nondirected, non-LOS) is considered to be the most flexible configuration in the literature [7].

\section{SIMULATION SET UP}

The simulation of various channel topologies have been carried out using Optiwave simulation tool as discussed below. 
Sharma, R. K. Kaushal, $\mathrm{H}$. Sharma, P. K.

\section{A. Directed (LOS) and Non-directed(LOS) Scenario}

The simulation setup for this scenario is shown in Fig. 2. It consists of a transmitter section which comprises of a pseudo random bit sequence (PRBS) generator with bit rate of $10 \mathrm{Mbps}$. The generated bits are encoded into NRZ-OOK format and given to a laser source with the transmit power of $-20 \mathrm{dBm}$ along with an optical power meter for normalization. The signal from the laser is then given to the optical channel i.e., free space medium. The distance between transmitter and receiver has been kept as 5 meters. The receiver consists of PIN photo diode, a low pass filter and an eye diagram BER analyser to visualize the results.

\section{B. Multi Beam Diffused Scenario}

The simulation set up for this scenario is described in Fig. 3. The transmitter side comprises of a PRBS generator with bit rate of $10 \mathrm{Mbps}$, the NRZOOK pulse generator for line coding and a laser source with the transmit power of $-20 \mathrm{dBm}$ along with an optical power meter. The receiver side consists of a PIN photo detector, a low pass filter, an eye diagram analyser for results analysis. Here the optical light is split into four equal beams to achieve multi beam diffused scenario. Thus the diffused multi beam is then given to free space optical channel. The quality of received signal and BER is analysed for various transmitter beam divergence angles.

The effect of transmitter beam divergence has been analysed for directed LOS, non-directed LOS and multi beam diffused topology. The analysis of simulated results are given in section IV.

\section{SIMULATION RESULTS AND ANALYSIS}

The simulation results of different topologies have been observed and analysed as per the following:

\section{A. Directed LOS Scenario}

In this scenario, the transmit power, bit rate and the distance between the transmitter and receiver has been kept constant as described in the simulation setup. The beam angle has been varied to observe the effect on BER, Q-factor and eye diagram which are shown in Fig. 4, 5 and 6 respectively.

The BER results are expressed in Fig. 4. Since the transmitter and receiver are in line of sight with extremely low beam divergence angle, the BER results reflect a very efficient communication link.

Journal of Technology Management for Growing Economies, Volume 6, Number 1, April 2015 
Q-factor indicates the quality of received signal. Higher the Q-factor, the better will be the quality of the received signal. From Fig. 5, it can be observed that signal quality deteriorates with increase in beam divergence angle.

The variance in eye diagram with respect to transmitter beam divergence angle is shown in Fig. 6. It can be seen that at narrow beam divergence, eye opening is wider indicating better noise margins and jitter. However as the beam divergence angle is increased, the eye opening gets narrower indicating lower noise margins and higher jitter.

In the simulation setup of directed LOS scenario, the results have been tabulated in the table I as follows.

Table 1: Results of Directed Los Scenario

\begin{tabular}{|c|c|c|c|}
\hline Beam Angle(mrad) & Log of BER & Q-factor & Eye Amplitude \\
\hline 2 & -1000 & 45 & 4.5 \\
\hline 4 & -280 & 35 & 3.3 \\
\hline 6 & -170 & 28 & 2.5 \\
\hline 8 & -110 & 22 & 2.0 \\
\hline 10 & -70 & 18 & 1.6 \\
\hline
\end{tabular}

Min. BER of LOS Channel , Bit Rate(10 MBPS)

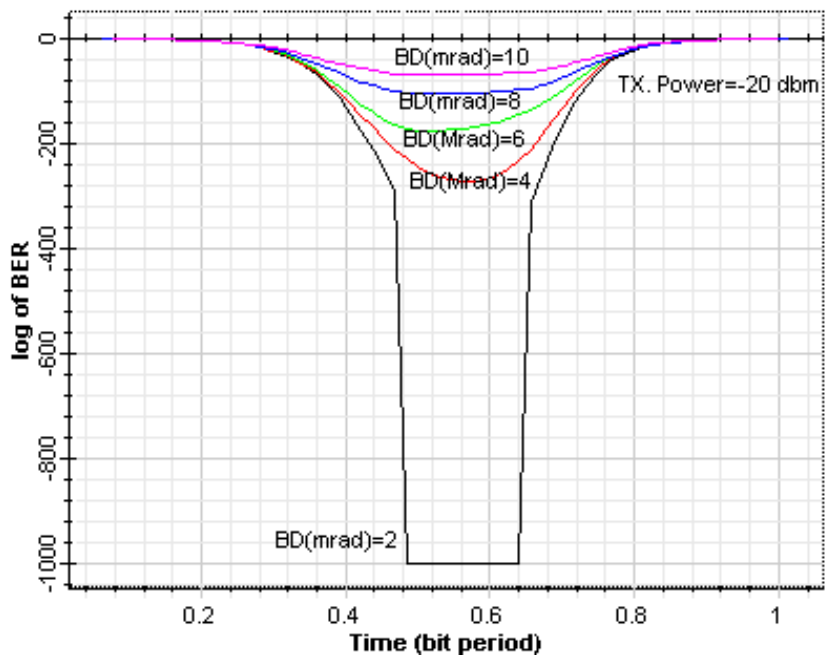

Figure 4: BER for directed LOS Scenario
Performance Analysis of Indoor 
Sharma, R. K. Kaushal, $\mathrm{H}$. Sharma, P. K.

\section{Q Factor for LOS Channelk, Bit Rate(10 MBPS)}

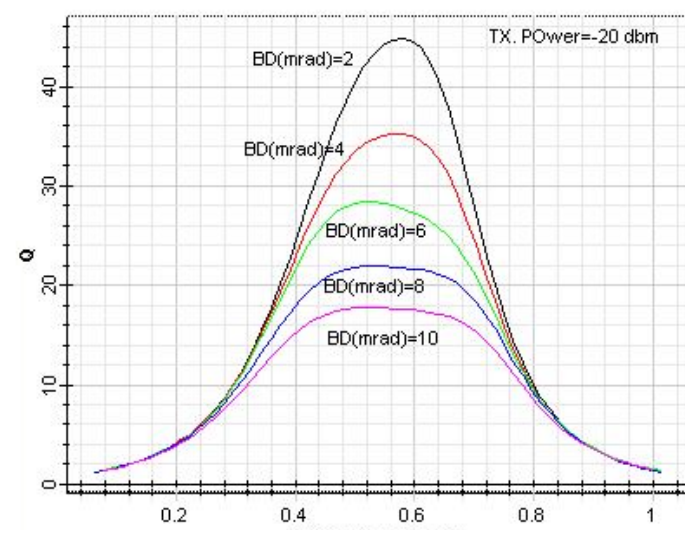

Figure 5: Q-factor for directed LOS Scenario

The results clearly show that directed LOS scenario is highly efficient at narrow beam divergence, however as the beam divergence angle is increased the SNR gets deteriorated significantly as can be seen from the respective plots.

\section{B. Results of Non-directed LOS scenario}

In this scenario also, the transmit power, bit rate and the distance between the transmitter and receiver has been kept constant as described in the simulation setup. The beam divergence angle has been varied to observe the effect on BER, Q-factor and eye diagram which are shown in Fig. 7, 8, 9 respectively.

Eye Diagram for LOS Channel , Bit Rate(10 MBPS)

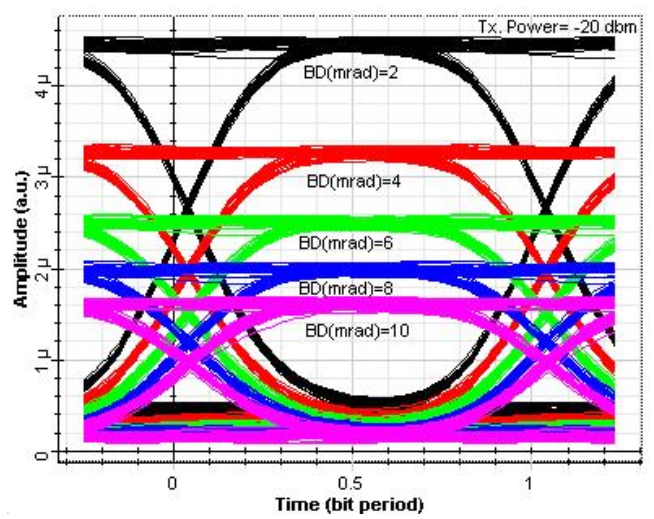

Figure 6: Eye diagram for directed LOS scenario

Journal of Technology Management for Growing Economies, Volume 6, Number 1, April 2015 
As the beam angle gets wider, the BER deteriorates more sharply as expressed in Fig. 7. This is because of the decreased radiation intensity per unit area as a result of wide coverage for a fixed Receiver field of view (FOV).
Performance

Analysis of Indoor

Min. BER for NON LOS Channel with wider Beam Divergence

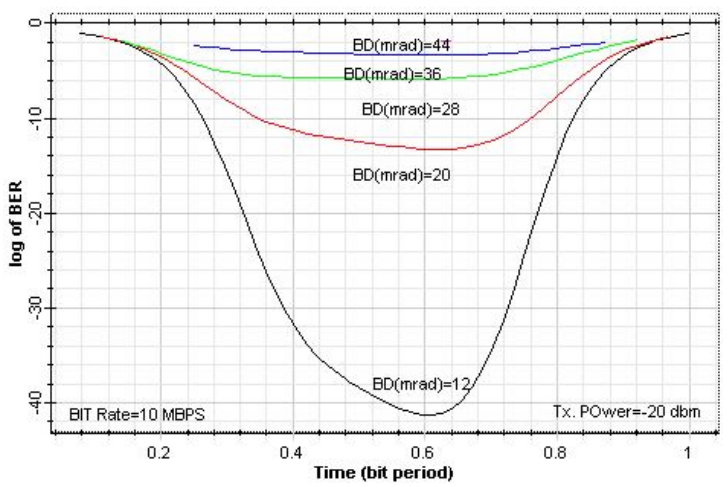

Figure 7: BER for non-directed LOS scenario

The Q- factor variance with respect to transmitter beam divergence angle is shown in Fig. 8. Here, it is clearly visible that signal quality continue to deteriorate with increased transmit beam angle.

Eye diagram performance for the non-directed LOS scenario is depicted in Fig. 9. The results show the reduced noise margins and hence reduced SNR affecting the system performance.

In the simulation set up of non-directed Los, the effects on system performance for wider beam divergence angles have

Q Factor for NON LOS Channel with wider Beam Divergence

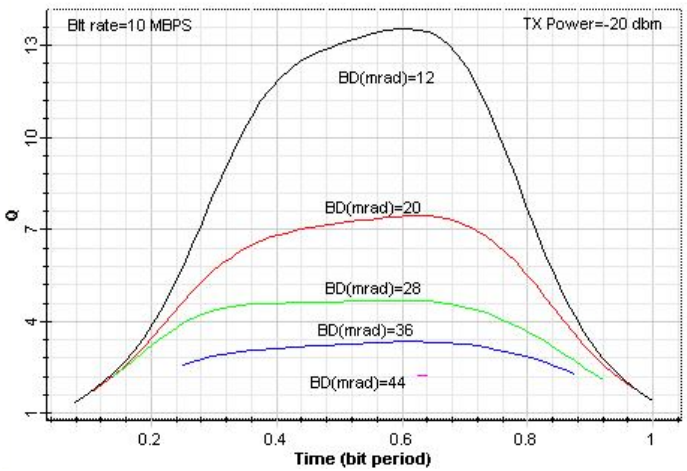

Figure 8: Q-factor for non- directed LOS scenario 
Sharma, R. K. Kaushal, $\mathrm{H}$. Sharma, P. K.
Eye Diagram for NON LOS channel with wider beam divergence angle

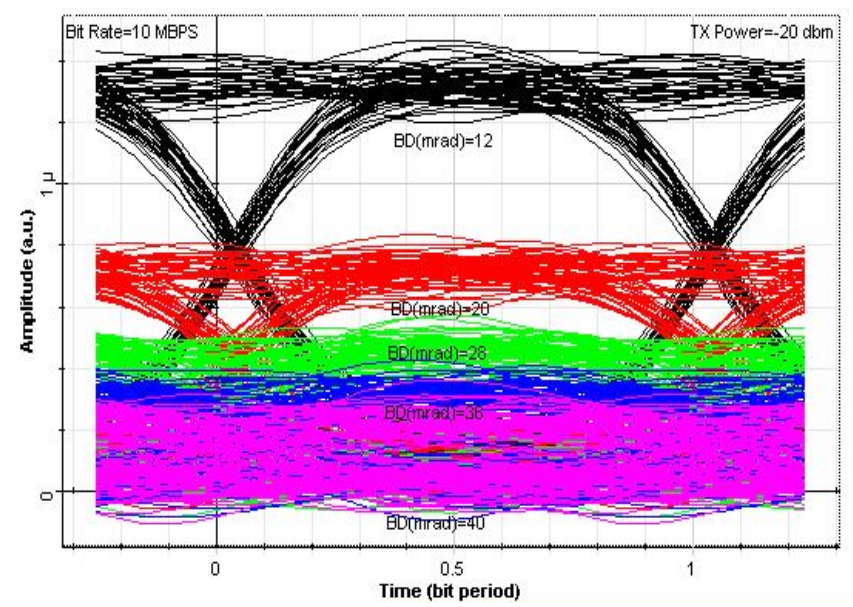

Figure 9: Eye diagram for non-directed LOS scenario

been analysed. The table II shows the results of variation of the beam divergence angle and its effect on the system performance. The nondirected topology seems to be more convenient to use particularly for mobile nodes but such topology may suffer higher transmission losses as can be observed from the respective plots.

Table 2: Results of Non-Directed Scenario

\begin{tabular}{|c|c|c|c|}
\hline $\begin{array}{c}\text { Beam } \\
\text { Angle(mrad) }\end{array}$ & Log of BER & Q-factor & Eye Amplitude \\
\hline 12 & -41 & 14.6 & 1.4 \\
\hline 20 & -13 & 7.6 & 0.8 \\
\hline 28 & -6 & 4.6 & 0.5 \\
\hline 36 & -3 & 3.4 & 0.4 \\
\hline 44 & 0 & 2.2 & 0.2 \\
\hline
\end{tabular}

\section{Results and analysis of multi beam diffused scenario}

In this simulation set up, the transmitted power has been split in to 4 multiple beams each of equal power in order to create the diffused link scenario. The results have been shown in Fig. 10, 11, 12 respectively. The bit rate has been kept at 10 MBPS. The BER results show an improvement in BER performance as compared to non-directed LOS scenario as expressed in Fig. 10. 
Min BER for MBD(1X4) System, Bit rate(10 MBPS)

Tx. Power $=-20 \mathrm{Dbm}$

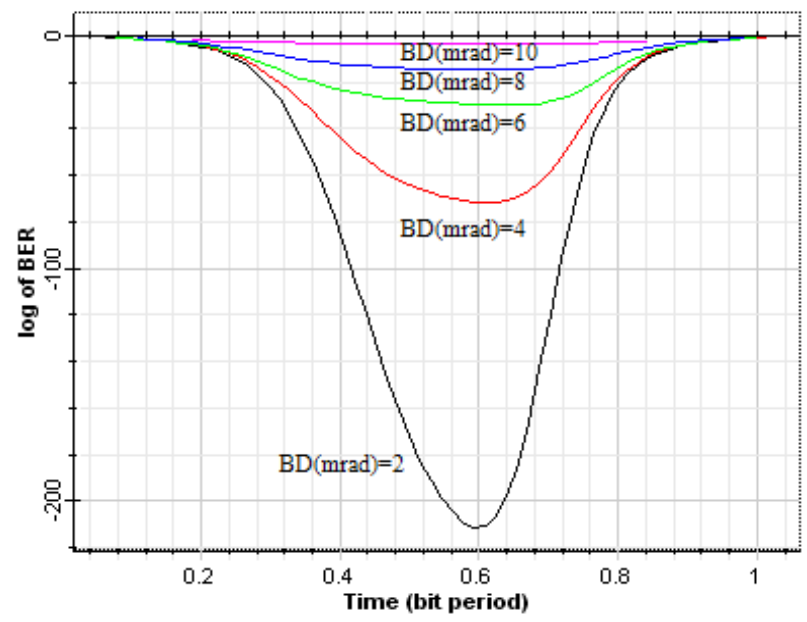

Figure 10: BER for multi beam diffused link scenario
Performance

Analysis of Indoor

The Q- factor results have also improved as compared to non-directed LOS scenario. Better SNR is achieved in this case with respect to non-directed LOS as presented in Fig. 11.

\section{$Q$ factor for MBD(1x4) System, Bit Rate $=10$ MBPS \\ Tx. Power $=-20 \mathrm{dBm}$}

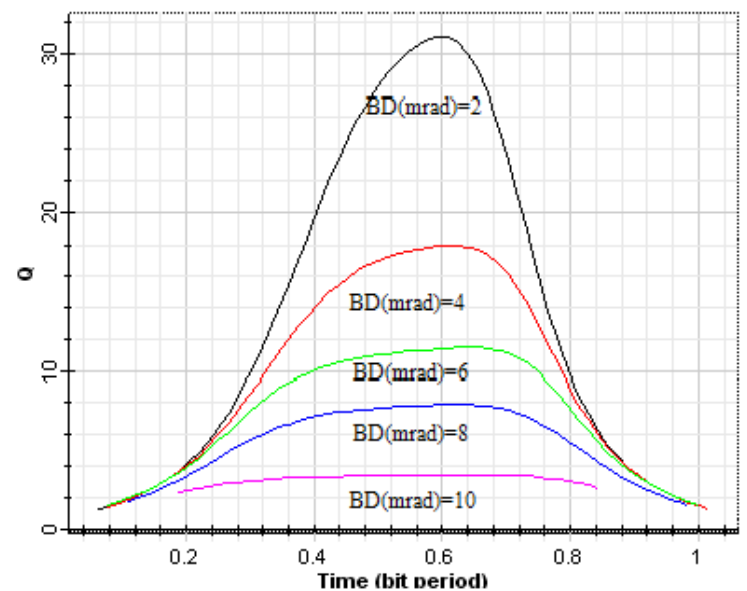

Figure 11: Q-factor for multi beam diffused link scenario

The eye diagram shows eye opening margins and respective jitter for varying beam divergence angles in this topology. This scenario appears to be most 
Sharma, R. K. Kaushal, $\mathrm{H}$. Sharma, P. K.

\section{4}

promising as this would lead to more robust channel formation since this is not confined and limited to degree of directionality between transmitter and receiver as was the case in LOS topology. The eye diagram results are expressed in Fig. 12.

\section{Eye Diagram for MBD(1x4) System, Bit rate= $10 \mathrm{MBPS}$}

Tx. power $=-\mathbf{2 0} \mathrm{dbm}$

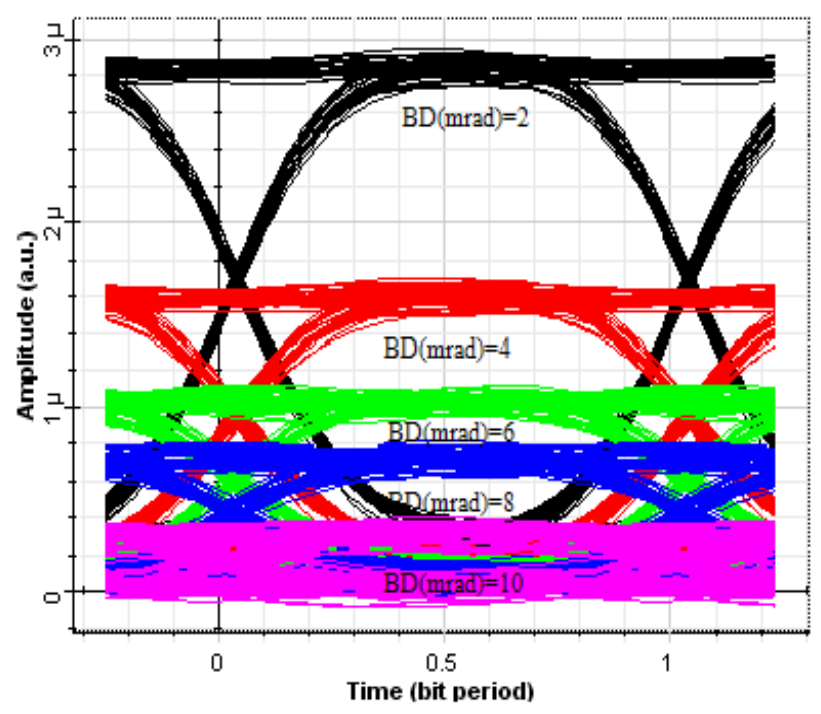

Figure 12: Eye diagram for multi beam diffused link scenario

The results of this scenario are presented in the Table III. These results are quite promising on all parameters as compared to non directed LOS system topology.

Table: 3 Results Of Multi Beam Diffused Link Scenario

\begin{tabular}{|c|c|c|c|}
\hline Beam Angle(mrad) & Log of BER & Q-factor & Eye Amplitude \\
\hline 2 & -220 & 31 & 2.8 \\
\hline 4 & -70 & 18 & 1.6 \\
\hline 6 & -30 & 12 & 1.0 \\
\hline 8 & -14 & 8 & 0.7 \\
\hline 10 & -4 & 4 & 0.3 \\
\hline
\end{tabular}

\section{CONCLUSIONS}

The compiled simulation results are shown in table IV which gives the comparison of various performance parameters for varying transmitter beam

Journal of Technology Management for Growing Economies, Volume 6, Number 1, April 2015 
divergence angle using various channel topology.

Table 4: The Comparison of Simulation Results

\begin{tabular}{|l|l|l|l|l|}
\hline Topology & $\begin{array}{l}\text { Beam } \\
\text { divergence } \\
\text { Angle(mrad) }\end{array}$ & Log of BER & Q-factor & $\begin{array}{l}\text { Eye } \\
\text { Amplitude }\end{array}$ \\
\hline & 2 & -1000 & 45 & 4.5 \\
\hline Directed & 4 & -280 & 35 & 3.3 \\
\hline LOS & 6 & -170 & 28 & 2.5 \\
\hline & 8 & -110 & 22 & 2.0 \\
\hline & 10 & -70 & 18 & 1.6 \\
\hline & 12 & -41 & 14.6 & 1.4 \\
\hline Non & 20 & -13 & 7.6 & 0.8 \\
\hline directed & 28 & -6 & 4.6 & 0.5 \\
\hline LOS & 36 & -3 & 3.4 & 0.4 \\
\hline & 44 & 0 & 2.2 & 0.2 \\
\hline & 2 & -220 & 31 & 2.8 \\
\hline Multi & 4 & -70 & 18 & 1.6 \\
\hline beam & 6 & -30 & 12 & 1.0 \\
\hline diffused & 8 & -14 & 8 & 0.7 \\
\hline & 10 & -4 & 4 & 0.3 \\
\hline
\end{tabular}

From the simulation results, it can be observed that the LOS channel topology being highly directional can be very attractive in terms of power efficiency but topology may not be very suitable for indoor mobility. It is subjected to interruptions due to shadow effect between transmitter and receiver. In this topology the transmitter and receiver are expected to be stationary which restricts mobility as such. In the non-directed LOS topology, the system performance suffers almost on all design parameters including high bit error rate and low SNR however, mobility in this case is high. The simulation results of the multi beam diffused link topology provides the better system performance in terms of high SNR and low bit error rate as compared to nondirected LOS topology. Such a link topology can be very effective and far more robust which allow the link to operate even in shadow effects or any obstruction that may occur between transmitter and receiver path. Hence such a link topology may be more suitable for indoor mobility.

\section{REFERENCES}

H. Elgala, R. Masleh and H. Hass (2011) "Indoor Optical Wireless Communication: Potential and State of the Art ", IEEE Communication Magazine. http://dx.doi.org/10.1109/ MCOM.2011.6011734

Journal of Technology Management for Growing Economies, Volume 6, Number 1, April 2015 
Sharma, R. K. Kaushal, $\mathrm{H}$. Sharma, P. K.
S. Jivkova and M. Kavehard (2000) "Multispot Diffusing Configuration for Wireless Infrared Access," IEEE Trans. on communications, Vol. 48:6, pp. 970-978. http://dx.doi. org/10.1109/26.848558.

P. Djahani, J.M. Kahn (1999) "Analysis of Infrared Wireless Links Employing Multibeam Transmitters and Imaging Diversity Receivers", Global Telecommunication Conference. http://dx.doi.org/10.1109/GLOCOM.1999.831689.

Ke. Wang, A. Nirmalathas (2011) "High-Speed Optical Wireless Systems for Indoor Applications", IEEE Phtonics Technology Letters, Vol. 23:8. http://dx.doi.org/10.1109/ LPT.2011.2113331.

J.M. Kahn, J.R. Barry (1997) "Wireless Infrared Communications," Proceedings of the IEEE, Vol. 85:2, pp. 265-298. http://dx.doi.org/10.1109/5.554222.

J.R. Barry, J.M. Kahn (1993) "Simulation of Multipath Impulse Response for Indoor Wireless Optical Channels," IEEE Journal on Selected Areas in Communications, Vol. 11:3. http:// dx.doi.org/10.1109/49.219552.

F.R. Gfeller, U. Bapst (1979) "Wireless In-House Data Communication Via Diffuse Infrared Radiation," Proceedings of the IEEE, Vol. 67:11, pp. 1474-1486. http://dx.doi.org/10.1109/ PROC.1979.11508. 